\title{
Simulation and experimental results of daily Cosmic Ray detection rate by an array including 5 detectors
}

\author{
Yousef Pezeshkian \\ Sahand University of Technology \\ Sahand New Town, Tabriz, East Azarbayjan, Iran \\ E-mail: yousef.pezeshkian@gmail.com \\ Mahmud Bahmanabadi \\ Sharif University of Technology \\ Tehran, Iran \\ E-mail: bahmanabadi@sharif.edu \\ Saba Mortazavi Moghaddam \\ Semnan University \\ Semnan, Iran \\ E-mail: mortazavimoghaddam@gmail.com

\section{Masoume Rezaie ${ }^{1}$} \\ Alborz Observatory \\ Sharif University of Technology, Tehran, Iran \\ E-mail: m. 83.rezaie@gmail.com
}

\begin{abstract}
Central cluster of Alborz-1 including 5 scintillation detectors in a pentagon configuration has been operated at the Sharif University of Technology campus. Data of 10 weeks operation is used to get the average daily Rate of Cosmic Ray detection. Comparing obtained experimental value of daily rate (2697) with that of simulation (5589) shows 107\% discrepancy. Modification of simulation code by considering detectors efficiency gives the daily rate of 3465 and reduces the discrepancy to about $29 \%$. Other parameters affecting daily rate in experiment and simulation are also discussed and relevant suggestions are given.
\end{abstract}

35th International Cosmic Ray Conference - ICRC2017

10-20 July, 2017

Bexco, Busan, Korea

${ }^{1}$ Speaker 


\section{Introduction}

The Alborz-I array with 20 scintillation detectors is designed to study cosmic rays (CRs) with energies around the knee at Sharif University of Technology, Tehran (35.72N 51.33E) at 1200 m a.s.l. [1]. Central cluster of Alborz-1 has been operating separately since October 2016 in order to perform several primary tests. Details of Albroz-1 configuration can be found on $[1,2]$. Data collected during the first 10 weeks of operation is used to measure daily rate of CR detection. During this period a negligible variation in ambient temperature is observed. Fluctuation in detection rate is given in Figure 1.

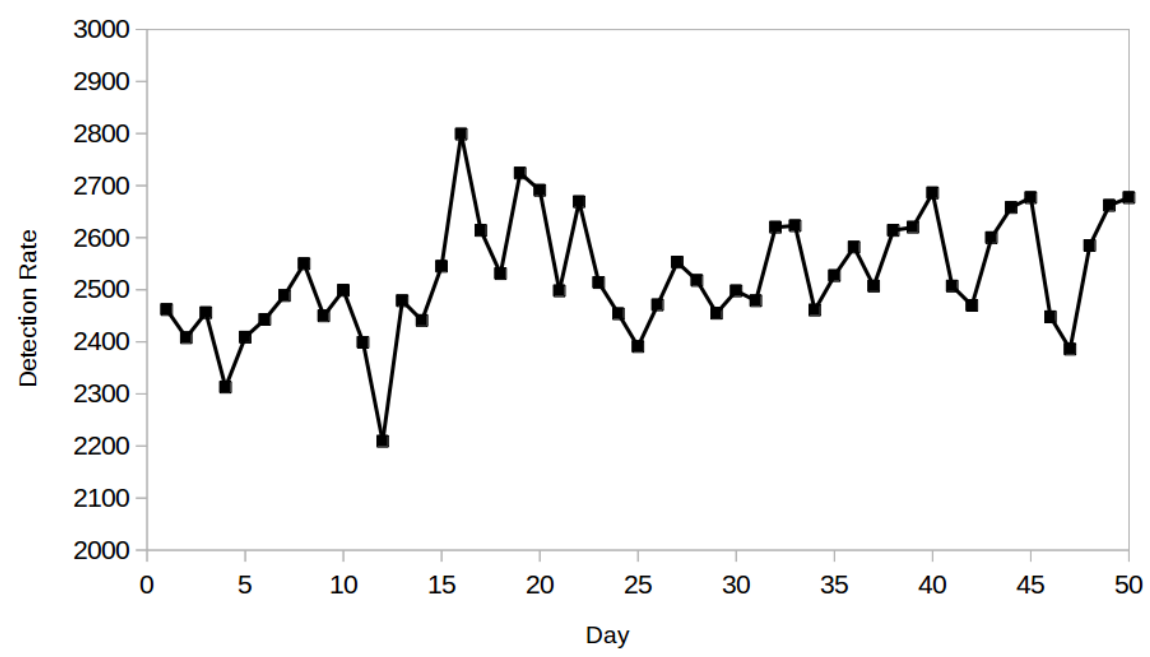

Figure 1 - Daily detection rate of CR

\section{Experiment}

In this experiment, 5 scintillation detectors (named det1, to det5 respectively) in corners of a pentagon with side length of $5 \mathrm{~m}$ (Figure 2) are installed on Alborz-1 location $1200 \mathrm{~m}$ a.s.l. Experiment started from 28th of October 2016 and data taking will be continued for about a year. We have used data of first 1200 hours of operation to deduce "daily CR detection rate".

Data acquisition is based on the coincidence method (for more detail see [4]). Coincidence between det 1 and det 2 triggers the array to record an event. A recorded event is considered an extensive air shower (not fake) when two conditions are satisfied: (1) all 4 coincidences are occured and (2) time lag of each one is placed in a bell curve of the spectrum, with a statistical error of about 2 ns. Events with zenith angles more that $45^{\circ}$ are excluded because of the walls around the array location.

Average number of daily events in which all 5 detectors are on, i.e. 4 coincidences are occurred, is about 4083. Filtering events with time lag out of bell curve of coincidence spectrum (width of $50 \mathrm{~ns}$ ) changes the number of events to 2697. Finally by limiting zenith angles less than $45^{\circ}$, average recorded CRs will be 2526 events each day, with standard deviation of 16 . 


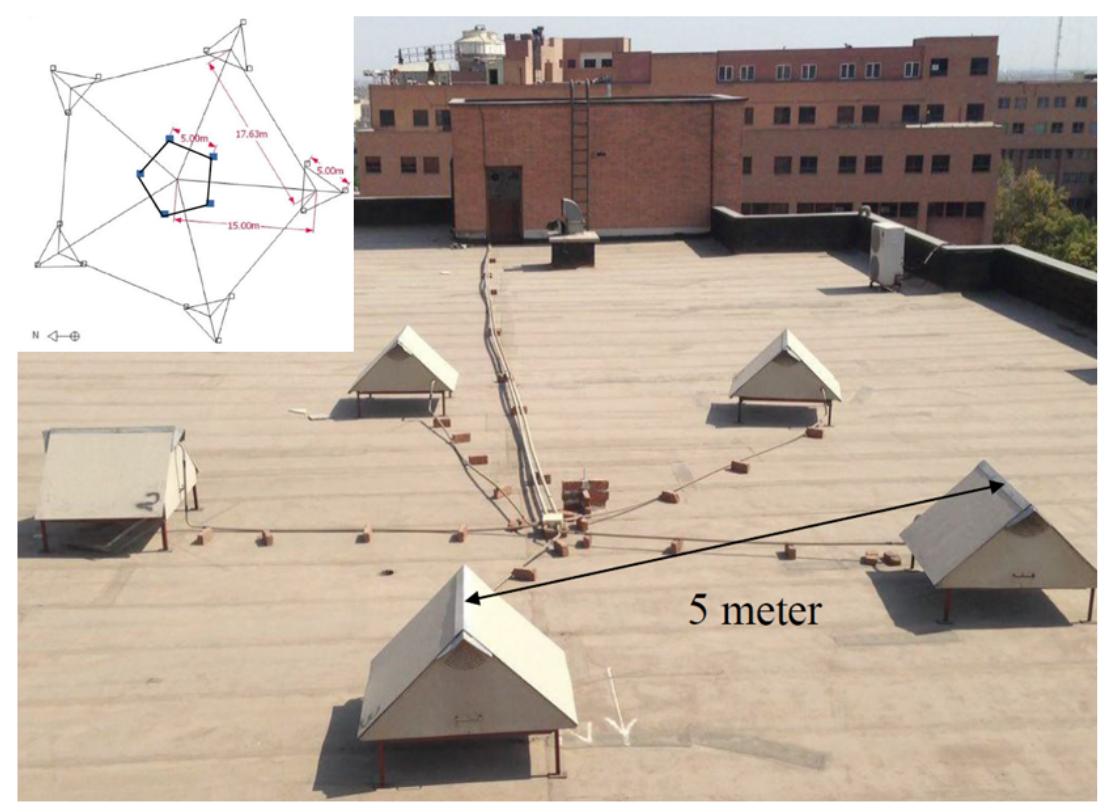

Figure 2 - Array located in Sharif University campus

\section{Simulation}

Daily rate of CR detection, for configuration of Alborz-1, is estimated by simulation in [2] and for trigger condition of " 5 out of 5 " in the central cluster corresponding result is 5645 (+948 -891). In order to be able to compare the value of simulation by experiment, we have to adjust zenith angle interval for both results. In the simulation zenith angles less than $60^{\circ}$ were included while in experiment we limited zenith angle to below $45^{\circ}$.

A simple simulation with CORSIKA data shows that about $1 \%$ of detected events in Alborz-1 array will have zenith angles between $45^{\circ}$ to $60^{\circ}$ (Fig. 3). By reducing $1 \%$ of events, 5589 events will remain for each day, which indicates $107 \%$ of discrepancy.

To uncover causes of this discrepancy different factors should be considered carefully. It should be noted that, a major part of observed discrepancy can be referred to detectors efficiency. In the simulation[2], detectors efficiency was not taken into account. While, it is shown in [3] that efficiency of Alborz-1 detectors is something less than 66\%.

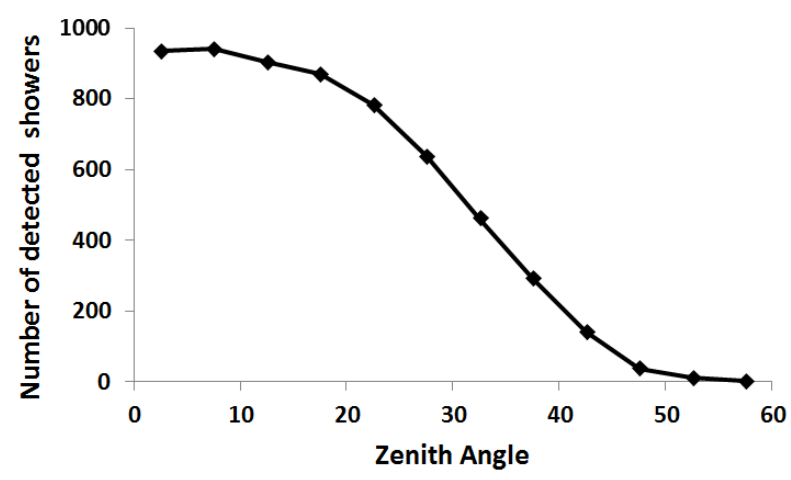

Figure 3 - Simulation result for dependence of CR detection rate on zenith angle. Less than $1 \%$ of detected events in Alborz-1 array will have zenith angle between $45^{\circ}$ to $60^{\circ}$ 
The simulation code is modified to consider detectors efficiency. We run the modified code for 2 energies ( $4 \mathrm{E} 14 \mathrm{eV}$ and $1 \mathrm{E} 15 \mathrm{eV}$ ) and the following results are obtained. In $4 \mathrm{E} 14 \mathrm{eV}$ number of daily detectable showers are reduced from 338 to 173 , which means that $49 \%$ of detected showers are diminished. Also in $1 \mathrm{E} 15 \mathrm{eV}$ number of daily detectable showers changed from 1221 to 792 , i.e. $35 \%$ of showers are not detected when detectors efficiency is $66 \%$.

By applying this trend to all energies, 38\% of total detectable showers will be diminished each day. So the value of daily detectable CRs given by simulation is 3465 (+582 -547).

Comparison of experimental value (2526 \pm 16$)$ and the simulation, we can conclude that the difference (939) is more than what we could relate it to the error bars (simul. 547, exp. 16) and still more investigation is necessary.

\section{Variation in efficiencies of different detectors}

Daily rate of CR detection, for configuration of Alborz-1, is estimated by simulation in [2] and for trigger condition of " 5 out of 5" in the central cluster corresponding result is 5645 (+948 -891). In order to be able to compare the value of simulation by experiment, we have to adjust zenith angle interval for both results. In the simulation zenith angles less than $60^{\circ}$ were included while in experiment we limited zenith angle to below $45^{\circ}$.

In [3], the efficiency of only 1 detector is measured and it is supposed that other detectors have the same efficiency. If the efficiencies of different detectors have large differences, it will affect the daily detection rate of CRs. To study the effect of variation in efficiencies experimentally, we have used data of recorded events to derive a simple test: The daily event rate with another trigger condition: " 4 out Of 5 " is obtained. When the coincidence of det 1 and det2 are used as trigger in experiment, there remains only 3 ways to choose 4 detectors as trigger detectors in offline data analysis (i.e. detectors 1234, 1235 and 1245). CRs daily detection rate for “4 out Of 5" is plotted in Fig. 4.

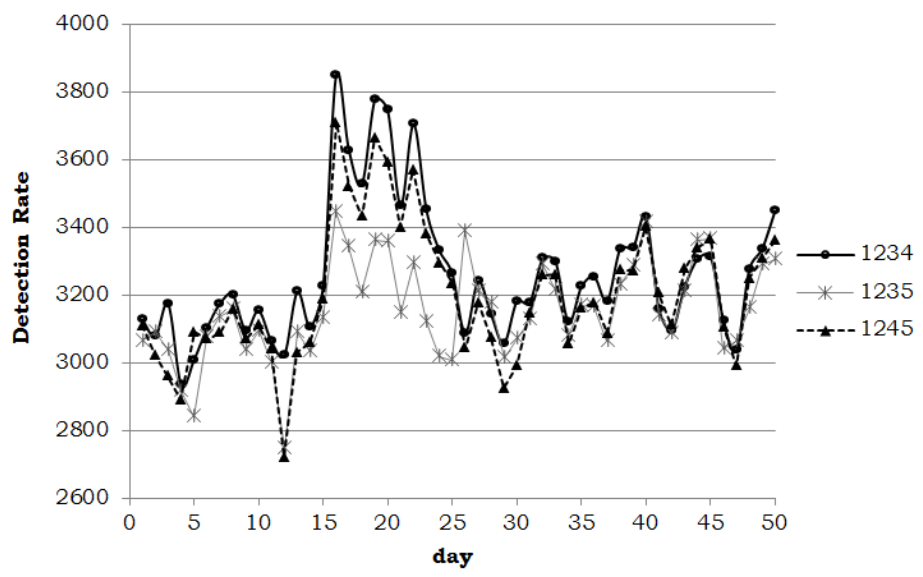

Figure 4 - Daily detection rate for trigger condition 4 out of 5.

Average daily detection rates for these 3 choices of triggering detectors 1234, 1235 and 1245 are 3263, 3154 and 3201 respectively. Variation in average values can be attributed to variation of efficiencies for different detectors. Overall effect of this error would be at most $2 \%$ change in experimental values ( $2 \%$ of 2526 is 51), which is small and negligible comparing the rate itself. 
There is still a possibility to have a considerable change in detection rate if instead of det1 and det 2 we use other detectors to trigger experiment. This needs further tests.

\section{Conclusion}

Detection rate of CRs by Alborz-1array extracted from data collected during 10 weeks of array operation is reported. The result was inconsitent with simulation published in [2]. Major part of inconsistency is ascribed to detectors efficiency. Modification of simulation code by adding detectors efficiency, reduces the inconsistency from $107 \%$ to $29 \%$. It should also be noted that reduction of detection rate strongly relies on primary CR energy (reduction changes from $49 \%$ to $35 \%$ in $\mathrm{CR}$ energies of $4 \mathrm{E} 14 \mathrm{eV}$ and $1 \mathrm{E} 15 \mathrm{eV}$ respectively). Therefore the simulation should be repeated for the rest of energies to get the better result.

Related important parameters in experiments could be (1) the applied filters in experiment which reduces detected events from 4083 to 2697 and (2) the discriminators threshold which are set to avoid noise and directly affect the threshold of detection energy for low energy particles. Further investigations is required to determine real share of these parameters. After recognising all sources of errors and their share on discrepancy, final calibration of simulation code according to experimental results will be possible.

\section{Acknowledgment}

We have to thank Mrs. Abdollahi to her useful comments to revise this paper.

\section{References}

[1] S. Abdollahi, et al., 34th International Cosmic Ray Conference (ICRC2015), vol. 34, 2015.

[2] S. Abdollahi, M. Bahmanabadi and Y. Pezeshkian; Astroparticle Physics 76 (2016) 1-8.

[3] Y. Pezeshkian, M. Bahmanabadi, M. Abbasian Motlagh and M. Rezaie, NIMA 773 (2015) 117-123.

[4] F. Sheidaei, et al., International Cosmic Ray Conference, vol. 5, pp. 909-912. 2008. 